\title{
Developing a replication of a wayfinding study From a large-scale real building to a virtual reality simulation
}

\section{Conference Paper}

Author(s):

Kuliga, Saskia; Charlton, James; Rohaidi, Hilal Fitri; Lu, Qian Qi Isaac; Hoelscher, Christoph; Joos, Michael

Publication date:

2020

Permanent link:

https://doi.org/10.3929/ethz-b-000418825

Rights / license:

In Copyright - Non-Commercial Use Permitted

Originally published in:

Lecture Notes in Computer Science 12162, https://doi.org/10.1007/978-3-030-57983-8_11 


\title{
Developing a replication of a wayfinding study From a large-scale real building to a virtual reality simulation
}

\author{
Saskia Kuliga ${ }^{1,3, *}$, James Charlton ${ }^{2}$, Hilal Fitri Rohaidi ${ }^{1}$, Lu Qian Qi Isaac ${ }^{1}$, Christoph \\ Hoelscher $^{1,3}$, and Michael Joos ${ }^{1}$ \\ 1 Singapore-ETH Centre, Future Cities Laboratory, Singapore \\ 2 Northumbria University, Newcastle, United Kingdom \\ 3 ETH Zurich, Zurich, Switzerland \\ *kuligaearch.ethz.ch
}

\begin{abstract}
Developing virtual reality (VR) simulations for replication of realworld studies in spatial cognition research is a tedious process, as numerous processes must be considered to achieve correspondence. In this chapter, we describe the development of a virtual model for a replication of a real-world study in the Seattle Central Library. The aim is to pragmatically report challenges and solutions in translating real-world conditions of complex and large-scale buildings into virtual reality simulations. For this aim, the chapter focuses on three steps for development: modelling the virtual environment, optimizing the performance, and designing the human-environment interaction.
\end{abstract}

Keywords: Virtual reality (VR, VE), Real environment (RE), Development, Replication, Comparison, Pre-occupancy evaluation, POE, Wayfinding

\section{Introduction}

Virtual Reality (VR) ensures high experimental control, exact measurements, and modifiable experimental manipulations. In the last decades, rapid innovations in hardand software boosted its adaptation in industry and research. One topic within VR research is examining the reproducibility of findings from the real world in virtual reality, using replication studies. A necessary prerequisite for replication studies is achieving comparability between the real, existing environment (RE) and the virtual simulation of the real environment (VE).

Since our team repeatedly faced similar challenges with translating replication studies into VR in the last decade, despite the rapidly evolving technologies, the goal of this chapter is deliberately pragmatic: to identify the most important challenges, so that other researchers can circumvent them. For this aim, the chapter describes considerations for replication studies in spatial cognition research. For spatial cognition, and particularly for wayfinding research, VR simulations are supportive when measuring how people acquire, represent, remember, and use spatial information, while controlling the contributing variables. 
This chapter specifically refers to the development of replicating a real-world wayfinding study in the Seattle Central Library in VR, and focuses on three key aspects: modelling the virtual environment, optimizing the performance, and designing the human-environment interactions, to answer the following question:

- Which considerations and technical steps does a research team have to overcome to translate conditions of a real-world study in a complex and large-scale building into a virtual reality simulation of the same building?

\section{Background}

Virtual reality (VR) existed for decades, before becoming widely affordable and accessible for consumers and researchers in the early 2010s (e.g., with the release of the Oculus DK1 headset that took advantage of developments in mobile processing power and hardware). However, the advantages and limitations of the potential VR had for researchers typically depended on the available technology at a given time. Nowadays, researchers across various disciplines integrate VR as a research tool; e.g., Web of Science indexes ca. 13.400 English documents with the term virtual reality in their title, and Google Scholar broadly estimates ca. 1.580.000 documents since 2017.

For the remainder of this chapter, the use of the term VR relates to a virtual model that is displayed on the lenses of a head-mounted display, while user movement and human-environment interactions are allowed. A head-mounted display shows a stereoscopic view on two screens, one for each eye, in real-time, while the user's dynamic viewpoints are generated based on their movement, and while the real-world surrounding the user is blocked out. For reading clarity, the chapter excludes discussing other devices, such as the CAVE, triple-screen, mobile screen, projection (etc.), and VR tools, such as hand- or full-body-tracking. As a general note about this: the choice of the devices and tools depends on the study's requirements, content, and context.

This chapter also focuses specifically on the use of VR for replicating a real-world study in the fields of spatial/architectural cognition and environmental psychology. When the term replication study is used, this means repeating a study from the realworld in a virtual simulation of the same real-world context, using the same methods, different experimenters, and different participants as in the real-world study. Replication studies provide further evidence that the initial study results with other participants and experimenters are generalizable while excluding extraneous variables.

Developing models that are spatially complex, multi-level, and large-scale typically remains a tedious process for researchers, often requiring skills in game design, modelling, and implementation in the game engine. Architects and urban planners generate computer models as part of their design and planning process, sometimes including VR representations for visualization purposes or evaluative walkthroughs [e.g., 1-4]. However, architecture models, often created for construction documentations, or still or animated visualizations, typically do not perform well in a game engine due to their high-polygon count/level of detail. Therefore, for such models to be used effectively with VR, one uses re-topology to transform high-polygon objects into simplified, low-poly versions, while keeping the objects' visual aesthetics. Further- 
more, studies aimed at comparing RE and VE correspondence typically utilize rather small-scale environments, such as single rooms, corridors, or one floor [e.g., 5-7], two to four floors [e.g., 8-11], or many floors yet small-scale models [e.g., 12]. Seldom are the VEs used in research simultaneously large-scale, multi-level, and spatially complex; often they rely on simplified detailing or interaction.

Throughout this chapter, we refer to the large-scale, multi-level Seattle Central Library (SCL) as example, as this renowned building holds a high spatial complexity (e.g., floors are rotated in both boundary and size), and covers 11 floors (two of which are only accessible for staff) on an area of $38.300 \mathrm{~m}^{2}$. A model of this particular building was required in order to replicate an earlier real-world wayfinding study within VR. The methods and materials of that study are documented in [13] and are not the focus of the current chapter. Here, we solely discuss the development of the VR replication. As such, this chapter describes steps in developing replication study in the field of spatial/architectural cognition, and specifically, in research about cognitive decision-making during wayfinding.

\section{Approach}

This section reports several technical and theoretical considerations for developing a replication of a real-world wayfinding study in VR.

\subsection{Modelling}

The key question in a replication study using VR is to what extent the environments correspond. Correspondence between a real and a virtual environment does not mean achieving the same numerical values in statistics. Rather, similarities in 'patterns' of results should become visible.

For instance, in a wayfinding study, corresponding result patterns can be identified if participants engage in similar route choices at similar locations as in the real world. Further behavioral metrics that might indicate corresponding result patterns between a real and a virtual building include hesitative pauses that occur at similar locations. These might reflect a lack of spatial or semantic information at a crucial decision point, or disorientation due to mismatches in wayfinders' mental representation of the space and the actual environment that surrounds them. Some of these metrics that are used in RE studies may correspond more than others, as the VE simulation by its nature differs from the real world; e.g., due to missing vestibular and proprioceptive feedback.

For replication studies, the virtual simulation does not necessarily need to rely on a photorealistic and highly-detailed model. However, it needs to be a similarly sufficient representation of the existing space. Thus, any cues that support perception of depth and distances, and that might affect spatial legibility, should be sufficiently translated from the existing building to the virtual simulation. Furthermore, the virtual simulation must represent the real environment's geometry, contrast/depth, and similar object types at similar locations, as well as sufficiently realistic interactions - all of 
these must be modeled to a degree that the virtual environment (VE) generates corresponding result patterns as the real environment (RE). Yet, full experiential and sensorial realism in this context might not be needed.

Achieving correspondence between real and virtual environments. Ensuring that the VR model has the exact geometries, spatial relations, sizes, boundaries (etc.), as the RE, is essential for a replication.

This means collecting extensive reference materials, e.g., floor plans, and photos and videos of all relevant objects, signage/directories, and materials/textures. Online videos can be consulted as further references. However, as real-world building users alter their environment over time, online materials are not as reliable as the researchers' materials collected during the time of the real-world study. For instance, in the SCL, facility managers arranged the bookshelves and sections differently throughout the years, thereby improving some of the previously identified wayfinding challenges. In other replication studies undertaken by the team, facility managers used colors to highlight different regions in the building or removed railings for crowd flow management. Given users adapt their spaces over time, it is useful to have reference material of the original study's date.

If geometries in the virtual model are only slightly inexact, accumulated complications can arise. For instance, in the SCL model, minor imprecisions accumulated to pretest participants virtually hitting their head when descending stairs. However, fixing this led to other accumulated challenges, e.g., signage being more visible from a given location than in the RE. To resolve both of these issues, manual checks of the virtual model were made against the supporting reference material.

On the one hand, even minor imprecisions in geometry affect visual accessibility, and as such can simplify wayfinding tasks; e.g., when participants in the VE acquire more environmental information than participants in the RE, because due to minor differences in geometry, they simply had more visual access. On the other hand, any changes in geometry, compared to the existing building, can also be used quite deliberately, e.g., for identifying design variants that can be tested in a systematic A/B design comparison. In replication studies, however, VR participants must be able to get exactly the same information as real-world participants at the same locations. Thus, any objects, including signage, must be placed at the same locations, unless one deliberately seeks to design variants of the RE.

Given that what constitutes a landmark is an arduous question, and wayfinders have different, and adaptable strategies, one could eternally model all possible features that might support wayfinding. In order to avoid doing this, researchers could focus on unique, salient features at relevant decision-points. Researchers ideally know, based on their real-world study, at which location in the building, and in which phase within wayfinding which decisions are possible, and how goal-relevant a particular environmental feature is. These features thus can be modelled accordingly.

At the same time, a specific feature that is relevant in one wayfinding task may not be relevant in another. For instance, in the SCL, bookshelf signs majorly informed participants' strategies in one task, but in other tasks they were less relevant, and hence could be omitted on these other floors. For instance, we modelled all signage 
(directories, blue pillars, large wall texts), but only modeled the bookshelf signs when they were relevant. A decision to omit such details must carefully be informed by the results of the study that is to be replicated. If it is unclear whether participants do need bookshelf signs in other wayfinding tasks, it is better to model these than to omit them.

Furthermore, small destinations might not trigger the recognition of a destination in VR. However, enlarging destinations would make them more salient in VR than in the RE. This might create differences in result patterns. Thus, in the SCL model, all six destinations were both of the same size and similar visual appearance as in the RE study. For instance, in one particular task, the participants' key strategy was using bookshelf information. However, due to the need to optimize the model for performance, we had to use the same bookshelf texture on all bookshelves. Upon starting the task in which bookshelf signs were relevant, pretest participants initially commented on their insecurity of not recognizing the destination, because all bookshelves looked similar. However, once they reached the correct area (a book in a particular section), pretest participants felt at ease. Here, the destination was recognizable by a higher-resolution texture (a series of specific books) within the standard bookshelf texture (a texture that looked like books but made it impossible to read titles once being closer to it). The specific clear texture of this smaller destination could only be seen once pretest participants were standing in front of the correct destination. The crucial point is that both the semantic category, as well as the size and visual salience of each destination in the VE should aim to closely match that of the study that is to be replicated [also cf. 13].

Achieving ambience and realism. Ambience modelling depends on the context and purpose of the study. In some studies, grey-scaled geometries are sufficient; e.g., when examining effects of geometry in isolation of other variables $[14,15]$. However, in studies that involve experiential measures, ambience or atmospheric cues are more important [8]. For the SCL replication, the same color scheme was used to that of the real building. For instance, the neon-yellow escalators were designed to be equally salient cues for wayfinding, as many participants in the RE study used them. Yet, while the all-red floor (level 4) was modelled using red ceilings, walls, and floors, allowing the level to be recognizable as that in the RE, in reality this level uses various shades of red. However, as described above, factors relevant for wayfinding must be included, whereas more irrelevant aspects are sufficient as approximate representations. In the pretesting, participants reported high immersion and realism, despite our model not being photorealistic.

In general, concerning the realism of ambience, it is technically possible to use high-polygon objects and achieve photorealistic graphics. However, this choice means more processing time to render each frame, and, hence, a performance decrease. Performance is best observed in the fluency of the frames per second, the speed at which the images move to generate the illusion of a changing environment. Thus, even with relatively few polygons and simple shaders, it is possible to create a suited and sufficient VR experience for a replication study (Fig. 1). 


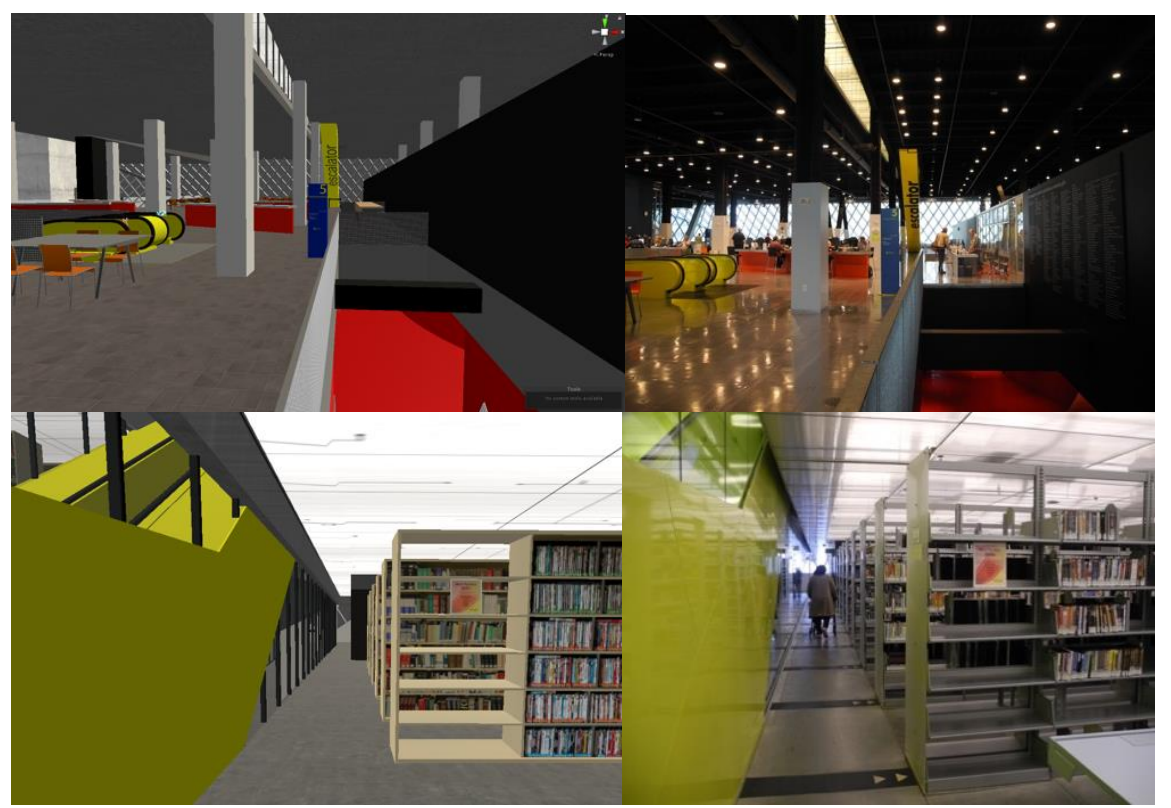

Fig. 1. Comparison of modelling between the virtual model (left) and existing building (right). Note: the camera lens has a smaller angle than the screenshot.

Materials such as wood, concrete, or glass that are existent in the real building can be approximated; i.e., they can be simplified to represent the material, but do not need to have the exact same color or texture. However, illuminance typically does influence wayfinding decisions. It has important effects for route choices; e.g., if a real-world environment contains dark hallways, participants may still walk into it, as long as it is not extreme. However, in VR, the effect of avoiding them may be more pronounced $[16,17]$. One solution is to illuminate all spaces equally, so that the route choices are based on the geometry and visibility.

In representing detailed objects with depth, such as the metallic grid railings and the highly complex façade of the SCL, it was considered that although creating a 3D geometry would look accurate, it would be time-consuming and detrimental to framerate performance, whereas using a texture was computationally fast but made the objects look flat. The adopted solution was to create an extrusion/displacement material that generates depth from a texture without the need to modify or model the object's geometry at the expense of a few additional draw calls (Fig. 2). 

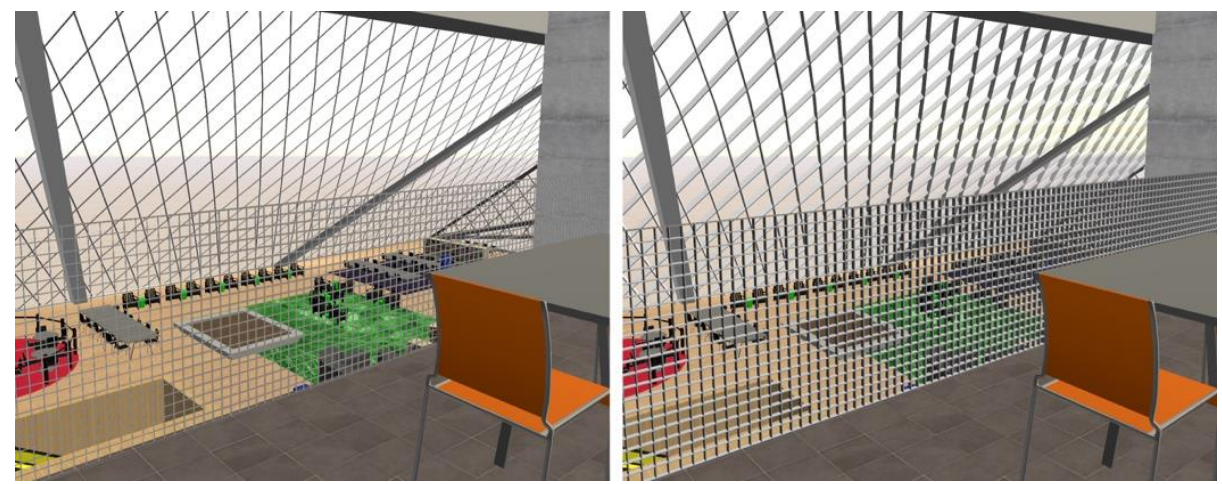

Fig. 2. SCL façade and railings: standard material (left), extrusion material (right)

Importing the model to the game engine. Technical import incompatibilities are common and not hard to solve, but researchers need to count in sufficient time for fine-tuning imports.

There are two common challenges when importing files from the initial modelling software (e.g., 3DsMax, Sketchup, Rhino, Blender) ${ }^{1}$ to the game engine (e.g., Unity, Unreal). One relates to the orientation of the model, the other relates to the orientation of the faces/polygons within a particular object.

The first challenge is due to different software adopting different $3 \mathrm{D}$ coordinate systems (i.e., left or right handedness) where the Z-axis may be pointing up, forward, or back. Generally, such issues can be dealt with as part of the import/export settings. However, in other cases, the orientations of the co-ordinates/Gizmo of objects themselves may need to integrated and resolved at a local level within the modelling software, before the model is exported. For instance, on one floor in the SCL model, chairs were initially imported rotated due to the local coordinate system of the chair not matching the global coordinate system of the overall model.

The second challenge relates to faces/polygons within an object being inverted or 'flipped', which can cause them to appear invisible within the game engine. It can be hard to identify this problem, as 3D modelling software by default shows both sides of the geometry, whereas game engines by default will only show the side with the polygon's normal pointing towards the camera, unless specified otherwise in the material. 3D modelers must therefore pay special attention to the orientation of the geometry's polygons and flip them accordingly, as otherwise objects may appear partially or entirely invisible in the game engine. For our team to detect such cases, we

\footnotetext{
${ }^{1}$ Readers who may wish to build upon the research discussed in this chapter may have access to alternative software, frameworks, data formats, etc. For instance, Maya and Blender are $3 \mathrm{D}$ modelling packages designed for animation and visual effects, whereas the native polygon modelling approach, texture mapping features, display functions, and the capability to support numerous file types and large-scale, detailed, and complex architectural models is well-established in 3Ds Max. However, the processes and guidelines discussed in this chapter would be relevant to most 3D modelling packages.
} 
created a custom material that draws every object in gray and 'flipped' polygons in bright yellow.

Another common challenge is flickering, called z-fighting, which occurs when two or more geometries share the same space/plane. In this case, the game engine does not know which geometry should be visible, and hence renders both alternately, causing the flickering effect. Here, a solution is to separate the offending polygons slightly from each other.

\subsection{Optimization}

After the aforementioned modelling process, ample time was spent on comparing the reference photos and videos with the virtual model, as well as on optimizing the performance.

Achieving the best performance in VR is paramount. Dropping below 90 frames per second (fps) typically causes discomfort/cybersickness and decreases immersion. We ran the model using Unity on an Intel Core i7-8700K CPU @3.7Ghz, 32GB RAM, NVIDIA GeForce GTX 1080 Ti GPU, displaying it on the HTC Vive Pro. However, our initial framerate was around 50fps despite running it on a powerful hardware.

Optimizing is a two-step iterative process that requires, first, to identify a performance bottleneck, and then, to apply a cost-cutting measure. There are tools available in Unity to profile the performance of the project and to identify bottlenecks. Initial profiling showed both high polygon count and material count; i.e., common indicators of low performance. A script that highlights clusters of high polygon and/or material density was created to help determine problem areas. By applying this script, the model's bookshelves, chairs, and escalators were found to be the culprits.

The easiest solution at this point was to simplify the geometry of those objects with high polygon count without compromising quality. Additionally, objects with multiple materials also saw their material count reduced: each bookshelf was reduced from 6148 triangles to 42 , and 37 materials were reduced to 1 (Fig. 3). The bookshelves and chairs also benefitted from enabling GPU instancing, which is a technique that draws multiple copies of the same mesh at once, only using a few draw calls. 

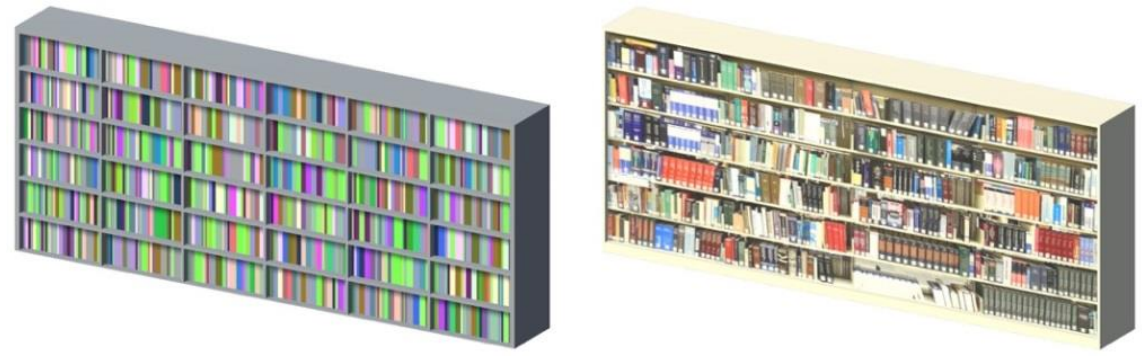

Fig. 3. Bookshelves with 6148 triangles and 37 materials (left) vs. 42 triangles, 1 material and 1 texture (right)

The second optimization solution was dynamically applied at runtime: to temporarily exclude floors that were not visible from the user's point of view from being processed by the GPU. For instance, the SCL's 'book spiral' on floors 6-10 would not be visible when participants were on floor one, and could be excluded. This required thorough testing to avoid floors suddenly disappearing.

\subsection{Interaction design}

Once the modelling and optimization stages are relatively complete, technical pretesting with participants is crucial. Such technical walkthroughs involve users interacting with the set-up, but without using the full experimental methods, such as offline questionnaires. This pretesting does not yet mean piloting. The aim of technical pretesting is to identify unforeseen human-environment interactions. For the technical pretesting, researchers should count in $20 \%$ of the time of the development duration.

During pretesting, unforeseen bugs can occur. For instance, in one SCL pretest round, a bug in a script caused participants to move to an entirely different floor after using the escalators. In one of our earlier VR replication studies, a participant was suddenly and involuntarily teleported to the roof of the building. Thus, in addition to standard protocols for informed consent including cybersickness information, when pretesting, researchers should a-priori inform participants about the possibility that such bugs can occur. Similarly, it should go without saying that, given participants using a headset are blind to their real-world surrounding, researchers should announce verbally if they intend to touch participants, e.g. when help is needed to adjust the headset. In brief, it is time researchers utilize standardized ethic protocols for VR research.

Movement. When replicating a real-world study, a key challenge is translating the movement of body parts, e.g. the hip rotation and head rotation, as well as simulating natural interactions that might occur in the real world.

Thus, one goal was to establish a synchrony between what the participant sees and their expectation what visually should happen. Another goal was to establish a synchrony between the participant's movement input (here: using the hands for move- 
ment in VR), and the speed, acceleration, and movement the system creates for the scene.

We used two controllers compatible with the HTC Vive Pro for tracking participants' hands, and a compatible belt for hip tracking. Participants swung their arms similarly to how they would do during natural walking. The speed of the swing determined the speed of their movement in VR.

The controllers provided information about their position and orientation, from which the scripts calculated speeds, acceleration, etc. Our controller input script had to distinguish arm swings from other types of arm movements, and at the same time account for different swing styles due to an individual's physique and walking habits. Once the swing speed was determined, signal processing was applied to smoothen sudden accelerations. The body direction could also be easily inferred from the two hand controllers, since both arms are generally swung in a mirrored fashion. Calculating the average of the two swing directions resulted in the forward vector. However, it was determined that installing a third tracker on the hip yielded a more accurate tracking. In sum, the particular setup of walking using the hands allowed participants to freely move in one direction while looking in another, to mimic how they would, for example, walk down a corridor while looking sideways for a book or sign.

For modelling collision boundaries, we defined the walkable space per floor, using the game engine's navigation mesh (Navmesh) feature, to set the walkable surfaces. For instance, objects such as tables, chairs, walls, and ceilings would be excluded as 'not walkable'. This is a standard procedure and explained in online tutorials for the game engine [e.g., 19].

Navigating through stairs or small steps initially resulted in an abrupt bumpy motion that was too jarring for VR. This was solved by replacing the affected navigation mesh area with a slope of equivalent angle that allowed the participant to traverse it smoothly and uniformly.

Some pretest participants additionally took small steps to navigate the VE, aside from swinging their arms, especially when changing directions. The small size of the real laboratory room presented a hazard for participants, despite the researcher closely watching their movements. Thus, we displayed a virtually visible marker of the center position in VR, so that participants could re-center themselves at any time. This procedure can also be done by letting participants press a button on the controller, but we chose not to risk disorientation by moving and/or rotating them virtually. We also marked the center space tactilely with a mat on the ground, so that participants would feel it when they were not centered.

Training room. Some researchers argue that a transition room from real to virtual is needed; e.g., a replica or virtual representation of the real laboratory room, from which participants could then transit to the main experiment [18]. In our view, a training room can equally serve as transition room, and it is furthermore useful to introduce the key interactions.

The key aim of a training room in the SCL (Fig. 4) is for participants to understand that they need to swing their arms to move in the virtual space and to practice how to navigate through the virtual environment. 


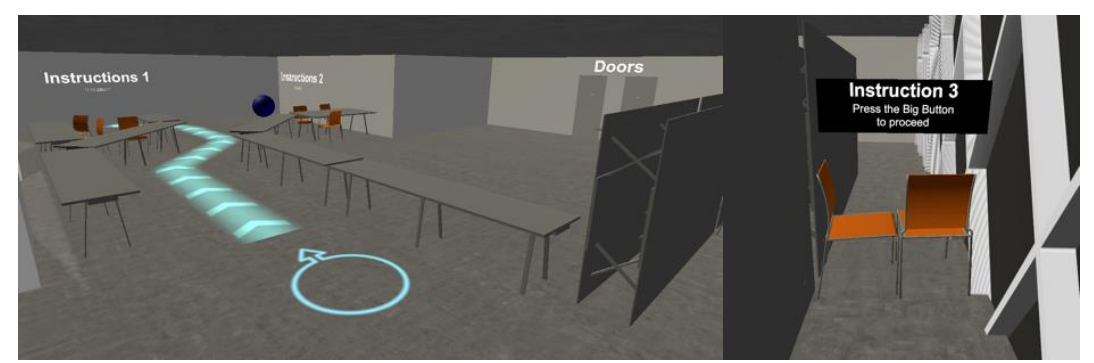

Fig. 4. Training room to learn interactions, including alignment circle and highlighted path (left) and discovery learning interactions (right)

First, participants followed a highlighted path on the ground that guided them to their first instructions on the opposite wall. The same highlighted path is used again later in the study when participants got lost or when they reached the time limit.

Second, after reading the initial instructions, participants moved freely through the room to discover more instructions. Both sets of instructions were intentionally in small letters so that participants had to navigate close to the locations and at the same time got used to finding small locations.

Third, participants had to find a certain door, but the shortest way was blocked with several tables and chairs. The aim was for them to learn how to avoid obstacles and navigate through narrow spaces. After the training room, participants started in the entrance area of the building and, following the highlighted path, had to walk to the first start point themselves.

In the training room, no objects were used that were potentially related to wayfinding in the main study. For instance, we chose not to explain the interactions with staircases and escalators, as this could potentially prime participants to prefer staircases and escalators in the main study.

Vertical level changes. For interaction design, researchers can rely on the interactions typically found in games. However, the more the interactions from the realworld are mimicked, the more likely one might find corresponding result patterns. For this aim, we kept game-like forms of interaction at a minimum. However, we created pop-up messages for discovery learning upon participants' first usage of escalators in the main study (Fig. 5). 


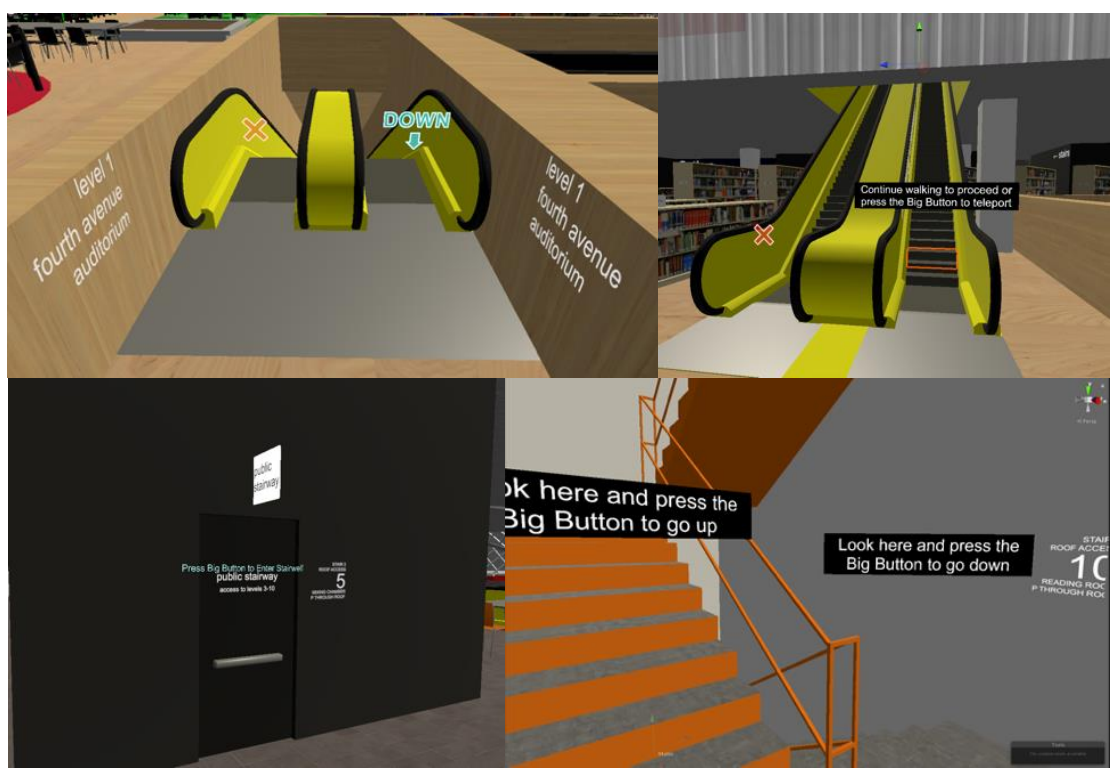

Fig. 5. Vertical level changes: interactions for using escalators and staircases

Escalators could be traversed either standing still while being moved or by walking and being moved at the same time. After receiving feedback from pretest participants complaining that the original staircases were static, we created a script to animate the steps to increase realism. If participants experienced any level of cybersickness, we also gave them the option to beam themselves to the other end of the escalator, rather than traversing it. In the latest pretest, five of seven participants stayed in VR for more than one hour and did not encounter cybersickness, while two pretest participants became cybersick after using the escalators and had to stop the study.

Staircases could be crossed by simply walking upwards. We could have changed this, but deliberately choose that participants could skip stairs by beaming only if they experienced cybersickness. Beaming would mean not acquiring or updating spatial knowledge, e.g., feedback about distances or vertical relations. In the SCL replication, this is an essential theoretical consideration, because several escalators bypass floors. Beaming would mean a lack of that information. This choice, however, has a limitation; i.e., some pretest participants got frustrated when they had to cross longer distances or several vertical changes. Given this effect was the same as in the realbuilding, we deliberately did could not optimize this interaction.

Whereas the escalators were not hard to develop, modelling the staircase user interaction required much development attention. The virtual space was small-size and there was a variety of interactions users could do: the system monitored whether a participant would want to be entering, make a choice to go up or down, or make a choice how to go up or down using either beam or walk. In the RE study, many participants were drawn more towards the visual saliency of the yellow escalators in the center of the floor, whereas the staircase was not visible from crucial decision points [cf. 13]. The usage of the stairs was equally rather low in the VR pretests. As such, 
the ratio of development time and participants' actual usage of vertical transfer options was higher/better for the escalators than for the staircases.

Standardizations of experimental procedures for the main study. During any study, there are participant-researcher interactions, such as providing instructions, that have to be carefully translated from the RE to the VE. For instance, in the real-world study in the SCL, a researcher walked the participant from the correct destination or the location of a failed task to the start point of the subsequent task.

While it is possible to model a simulated agent to behave like a researcher, it costs significant time and effort for the development team. One has to design how to treat situations when the virtual researcher stands in the way of the participant, or when the virtual researcher walks too fast and far away from them. A more robust solution is to display a highlighted path on the floor and instruct participants to follow the line that leads to the next task's start location. Thus, for the SCL, for the case that a participant failed to find the task destination, the shortest way from their current position to the next task's start location was dynamically calculated and displayed. A script was written to calculate the shortest path either to the end destination when it was on the same floor or to the elevators otherwise, to follow the same protocol as the real-life experiment. Once we implemented the highlighted path, however, some participants started looking at signage/directories, or deviated slightly to have more visual accessibility to the space around them. In the real-world, due to the real researcher walking in front of participants, this did not happen. Thus, for the VE, the researcher had to carefully instruct and observe participants.

Another implementation we used in earlier VR-replication studies was a standardization of the researcher's instructions, using a prerecorded voice. The reason is twofold: first, the voice of a real researcher can come from a different direction a participant expects while being immersed in VR. Second, a voice makes sure instructions are standardized across participants. However, the trade-off is that one needs to expect a range of participant-researcher interactions. In complex studies, this can lead to a mix of, e.g., having a virtual researcher, a prerecorded voice, and the real researcher intervening when needed. Thus, we did not use the instruction voice in the SCL study.

\section{Tools for the researcher.}

User interface. The researcher needed a user interface for behavioral mapping. In its basics, this meant entering a participant ID and order of the wayfinding task, and a button to start and finish tasks, as well as the entire study. In its extended use case, however, the user interface for the researcher must allow orchestrating a set of actions at the right time (Fig. 6, left). 


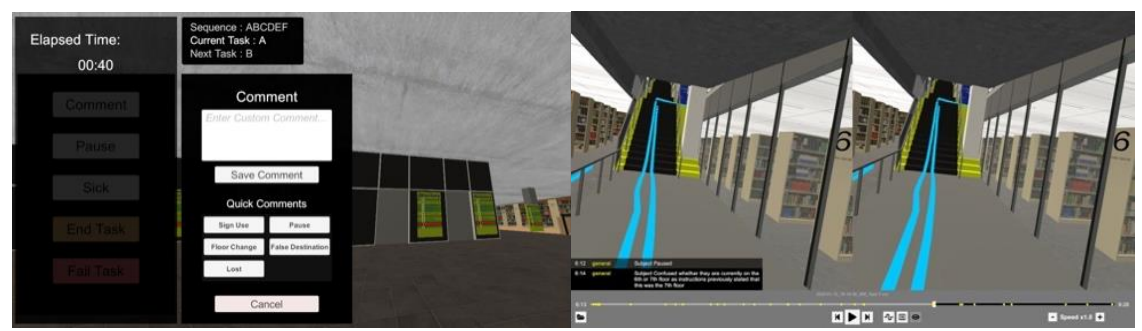

Fig. 6. Interface for the researcher during the study (left), and replay tool standalone to examine data visually (right)

The user interface depends on the context of the study. In our case, the researcher needed a start-task button, to activate any desired wayfinding task, and an end button to end that task. For start task, the researcher needed participants to be at a specific location and looking into a specific direction, so that the initial view and potential movement direction were standardized across participants. One solution for aligning participants was that participants moved to a designated and visually marked spot they could recognize. For instance, we implemented a circle with a directional arrow, and participants had to align themselves in the center, facing into the direction of the arrow. One can also arrange a button press to align participants automatically, but we chose not to align participants automatically, in order to avoid disorienting them.

Once participants were aligned in the way the study design needed, their movement was blocked and the researcher gave the task instructions by reading them out aloud. The system recorded and logged movement in this instruction phase, because participants might already look around for self-localization; e.g., turn their head to examine spatial openings or nearby signage. After the instructions, the researcher needed another button that disabled the movement block and recorded the start time of the movement, stopping when participants either reached the correct destination, or failed a task. We labeled this a 'go'-button, because we said "go" to the participant to start each wayfinding task - it can obviously be labeled differently. The key point is to be able to separate the durations of each of the wayfinding tasks in the study, as this will support data preparation and analysis.

During the main study, the researcher could manually take notes using a 'comment'-button or press a subset of buttons to record events (i.e., sign use, pause within a task, floor change, lost, false destination), or pause the entire study, or abort or pause the study due to cybersickness. This set-up was inspired by and developed further based on the app Peoplewatcher [19]. We did not make the user interface more detailed (i.e., more complicated to the researcher), because the researcher was already highly engaged with watching the participant and the screen with the participant's view. Once a participant was in the correct destination area, the button 'end task' was highlighted, so that the researcher would always be aware. This highlight was also triggered when the time limit had been reached. 
Visual inspection and analysis. How one organizes the output files with the recorded data depends on the study. In our case, we created one recording per task (the training room being the first one), with each recording containing two files:

- Participant movement: included timestamps and coordinates of participant body and head position and rotation.

- Events: included timestamps, researcher notes, and automated events such as 'floor change.'

The researcher notes were entered during the task either manually, in the user interface for the researcher (e.g., 'participant states it is logical to find the destination using the directories') or by pressing a button with predefined notes (e.g., 'sign use').

We built a separate standalone application where the researcher could replay and inspect these data recordings. Both recording files used the same Unix timestamp epoch to be able to synchronize movements with events and visualize them simultaneously. All movement paths and events were displayed similarly to video player, with the ability to pause, resume, scrub, jump to a particular time, and increase or decrease playback speed (refer back to Fig. 6, right).

With the described set-up, gaze patterns could not be calculated in real-time during a wayfinding task, as this would reduce performance. Given gaze data could only be analyzed post-hoc, the workaround was using the replay standalone to identify when relevant objects enter the center of the field of view of the participant. This setup did not allow classical eye-tracking data but approximated the direction participants looked at. In future work, we plan to use the HTC Vive Pro Eye that allows tracking eye gaze data. Such data can post-hoc be analyzed with a script. For this aim, some objects may need to be categorized beforehand.

\section{Discussion}

This chapter highlighted theoretical and technical steps we took while translating a real-world study from a complex, large-scale, existing building to a virtual reality simulation of the same building.

To test the comparability, researchers need to first evaluate the virtual replica of the original building as a baseline, and then test variants of the original spatial layout or experimental procedures. Examples of spatial variants are adding an atrium or widening staircases to enhance visibility and to increase available spatial information [8]. It could also be more subtle changes in local features, such as adding railings as movement barriers. Examples of changes in the experimental procedure are varying the wording in the instructions, to examine how the study's instructions might have triggered certain wayfinding strategies or participant expectations.

Given its potential for evaluating redesigns, VR simulations are useful tools for urban planners and architects who seek to vary design ideas they have at an early stage. This is called pre-occupancy evaluation, an evaluation before the building's or area's construction $[2,20]$. The physical models that architects regularly create during planning and design are typically scaled down in their sizes and do not contain a high 
level of detail. Hence, they may provide different experiences than the later constructed building. The potential of virtual reality is that it allows a $3 \mathrm{D}$ immersion into the perspective of a future building user. Although architects are used to mentally translating allocentric (top-down) floor plan views to egocentric views, earlier collaborations indicated that it is challenging to immerse themselves into the users' perspective [21]. As such, VR can be a useful evaluation pre-occupancy evaluation tool [8], e.g., for building and urban planners, who can wear a headset to quickly evaluate certain perspectives in their design from a 3D user perspective, or conduct expert walkthroughs with other experts and stakeholders, or immerse users for controlled $\mathrm{A} / \mathrm{B}$ comparisons or rapid prototyping.

All of these use cases require developing and choosing a degree of realism, optimizing performance if a model is highly detailed, and thinking about modelling human-environment interactions, such as the ones described in this chapter. Grey-scaled geometry models may serve the purpose of understanding effects of sizes and forms, and level of detail can be added incrementally. However, the level of detail, as we discussed in this chapter, should fit the purpose. If the aim is a replication study that included experiential and emotional measures, a degree of realism is a prerequisite.

As limitation of this work, for achieving proper VR performance with the available hardware, our virtual environment had to remain devoid of other people. We excluded virtually simulated agents (controlled by algorithms), avatars (controlled by a human), and wizard-of-Oz systems (seemingly controlled by an algorithm but controlled by a human). As such, we cannot elaborate on adding virtual agents to behave like humans in this model, as performance would drop again. One could argue that a building or urban environment evaluation in VR remains limited when being devoid of people, as life unfolds in these spaces by human-environment interactions. However, the benefit of using an empty virtual model is that one tests the impact of the spatial and semantic organization in isolation of social factors. Recent studies start integrating virtual agents in different VR contexts, e.g., to measure social effects or in pre-occupancy evaluations $[10,21,23]$.

In future work, we will not only collect data on the correspondence between result patterns in the virtual environment, as compared to our real-world study, but furthermore test architectural redesigns and spatial variants that might improve the identified wayfinding challenges. We also might vary our wording in the instructions, or on signage, to test how semantics influenced the wayfinding strategies. During the pretests, we overheard participants informally mentioning similar wayfinding strategies and spatial/semantic difficulties in the SCL. Naturally, we need proper pilot-testing and the main study to understand these interactions in a more nuanced fashion. However, given the informal comments in the pretests, we are optimistic to expect corresponding result patterns between the real and virtual building; i.e., to find only limited differences between the real and virtual environments, once we conduct the main study. 


\section{Conclusions}

The aim of this chapter was pragmatic rather than theoretical. Naturally, in other settings, other solutions than described the ones in this chapter are advisable. We aimed at describing the particular solutions we developed, to encourage other researchers, who are developing replications of real-world studies in virtual reality, to share their implementation solutions between laboratories.

\section{References}

1. Schneider, S., Kuliga, S., Weiser, R., Kammler, O., \& Fuchkina, E. (2018). VREVAL-A BIM-based Framework for User-centered Evaluation of Complex Buildings in Virtual Environments. In Anetta Kepczynska-Walczak, and Sebastian Bialkowski (Eds.), Computing for a better tomorrow - Proceedings of the 36th eCAADe Conference - Volume 2, Lodz University of Technology, Lodz: Poland, pp. 833-842. Retrieved on June 22, 2020 via http://papers.cumincad.org/data/works/att/ecaade2018_361.pdf

2. Kuliga, S. (2016). Evaluating user experience and wayfinding behaviour in complex, architectural environments - towards a user-centred approach of building usability, Doctoral dissertation, University of Freiburg, Germany. Retrieved on June, 23, 2020 via https://freidok.uni-freiburg.de/data/11386

3. Franz, G. (2005). An empirical approach to the experience of architectural space. Doctoral Dissertation, Max Planck Institute for Biological Cybernetics Tübingen and Bauhaus University Weimar, Germany, Logos-Verlag. Retrieved on June, 23, 2020 via via http://citeseerx.ist.psu.edu/viewdoc/download?doi=10.1.1.131.3592\&rep=rep1\&type= pdf

4. Westerdahl, B., Suneson, K., Wernemyr, C., Roupé, M., Johansson, M., \& Allwood, C. M. (2006). Users' evaluation of a virtual reality architectural model compared with the experience of the completed building. Automation in construction, 15(2), 150-165. DOI: 10.1016/j.autcon.2005.02.010

5. Chamilothori, K., Wienold, J., \& Andersen, M. (2019). Adequacy of immersive virtual reality for the perception of daylit spaces: Comparison of real and virtual environments. Leukos, 15(3), 203-226. DOI: 10.1080/15502724.2017.1404918

6. de Kort, Y. A. D., Ijsselsteijn, W. A., Kooijman, J., \& Schuurmans, Y. (2003). Virtual laboratories: Comparability of real and virtual environments for environmental psychology. Presence: Teleoperators \& Virtual Environments, 12(4), 360-373. DOI: $10.1162 / 105474603322391604$

7. Süzer, Ö. K., \& Olguntürk, N. (2018). The aid of colour on visuospatial navigation of elderly people in a virtual polyclinic environment. Color Research \& Application, 43(6), 872-884. DOI: $10.1002 / \mathrm{col} .22272$

8. Kuliga, S. F., Thrash, T., Dalton, R. C., \& Hölscher, C. (2015). Virtual reality as an empirical research tool-Exploring user experience in a real building and a corresponding virtual model. Computers, environment and urban systems, 54, 363-375. DOI: 10.1016/j.compenvurbsys.2015.09.006

9. Lazaridou, A., \& Psarra, S. (2017). Spatial navigation in real and virtual multi-level museums. In Teresa Heitor, Miguel Serra, João Pinelo Silva, Maria Bacharel, Luisa Cannas da Silva (Eds.), Proceedings of the 11th International Space Syntax Symposium, Lisbon, Por- 
tugal: Instituto Superior Tecnico, 14, pp. 1-18. Retrieved on June 22, 2020 via https://discovery.ucl.ac.uk/10038355/1/Lazaridou_Psarra.pdf

10. Li, H., Thrash, T., Hölscher, C., \& Schinazi, V. R. (2019). The effect of crowdedness on human wayfinding and locomotion in a multi-level virtual shopping mall. Journal of Environmental Psychology, 65, 101320. DOI: 10.1016/j.jenvp.2019.101320

11. Münzer, S., \& Zadeh, M. V. (2016). Acquisition of spatial knowledge through selfdirected interaction with a virtual model of a multi-level building: Effects of training and individual differences. Computers in Human Behavior, 64, 191-205. DOI: 10.1016/j.chb.2016.06.047

12. Andrée, K., Nilsson, D., \& Eriksson, J. (2016). Evacuation experiments in a virtual reality high-rise building: exit choice and waiting time for evacuation elevators. Fire and Materials, 40(4), 554-567. DOI: 10.1002/fam.2310

13. Kuliga, S. F., Nelligan, B., Dalton, R. C., Marchette, S., Shelton, A. L., Carlson, L., \& Hölscher, C. (2019). Exploring individual differences and building complexity in Wayfinding: The case of the seattle central library. Environment and Behavior, 51(5), 622665. DOI: $10.1177 / 0013916519836149$

14. Bielik, M., Schneider, S., Kuliga, S., Griego, D., Ojha, V., König, R., ... \& Donath, D. (2019). Examining trade-offs between social, psychological, and energy potential of urban form. ISPRS International Journal of Geo-Information, 8(2), 52, 1-31. DOI: 10.3390/ijgi8020052

15. Von Stuelpnagel, R., Kuliga, S., Büchner, S. J., \& Holscher, C. (2014). Supra-individual consistencies in navigator-driven landmark placement for spatial learning. In Proceedings of the Annual Meeting of the Cognitive Science Society, 36, 1706-1711. Retrieved on June 22, 2020 via https://pdfs.semanticscholar.org/0347/dd50c3ee5ddd56f64e52d5a25f4843f8e2b1.pdf

16. Langenfeld, V., Rist, M., Dalton, R. C., \& Hölscher, C. (2013). What space syntax does not know: movement triggers beyond integration. In Y. O. Kim, H. T. Park, \& K. W. Seo (Eds.), Proceedings of the 9th International Space Syntax Symposium, Seoul, South- Korea. Sejong University: Seoul, 076:1-18. Retrieved on June 22, 2020 via http://nrl.northumbria.ac.uk/id/eprint/17162/1/langenfeld_rist_dalton_holscher.pdf

17. Toet, A., Van Welie, M., \& Houtkamp, J. (2009). Is a dark virtual environment scary? Cyberpsychology and Behavior, 12(4), 363-371. DOI: 10.1089/cpb.2008.0293

18. Steinicke, F.; Bruder, G.; Hinrichs, K.; Steed, A. (2010). Gradual transitions and their effects on presence and distance estimation. Computer Graphics, 34, 26-33. DOI: 10.1016/j.cag.2009.12.003

19. Dalton, N., Dalton, R. C., \& Hölscher, C. (2015). People Watcher: an app to record and analyzing spatial behavior of ubiquitous interaction technologies. In S. Gehring \& A. Krüger (Eds.), Proceedings of the 4th International Symposium on Pervasive Displays, (pp. 1-6). DOI: $10.1145 / 2757710.2757714$

20. Kuliga, S., Weiser, R., Falke, S., \& Schneider, S. (2018). Nutzerzentrierte Gebäudeevaluation mittels Virtueller Realität. EI - Der Eisenbahningenieur: Internationale Fachzeitschrift für Schienenverkehr \& Technik, 4/18, Verband Deutscher Eisenbahn-Ingenieure e.V. (VDEI), Frankfurt/Main, 56-60; Retrieved on June 22, 2020 via https://www.researchgate.net/publication/324057127_Nutzerzentrierte_Gebaudeevaluation _mittels_Virtueller_Realitat

21. Hölscher, C., Brösamle, M., \& Dalton, R. C. (2010). On the role of spatial analysis in design synthesis: the case of wayfinding. NSF International Workshop on Studying Visual and Spatial Reasoning for Design Creativity (SDC'10), France, 1-5. Retrieved on June 22, 
2020

via

https://pdfs.semanticscholar.org/3c97/a9a6f9e50bcbe1407d6bafc270a68c56b732.pdf

22. Dubey, R. K., Kapadia, M., Thrash, T., Schinazi, V. R., \& Hoelscher, C. (2017). Towards an Information-Theoretic Framework for Quantifying Wayfinding Information in Virtual Environments. In CAID@ IJCAI (pp. 40-46). Retrieved on June 22, 2020 via https://www.research-collection.ethz.ch/handle/20.500.11850/229224.1

Author notes: The authors thank Dan Baird for supporting the development of signage for the VR model. The first author contributed to this chapter during a postdoc fellowship of the German Academic Exchange Service (DAAD), at the Future Cities Laboratory, Singapore-ETH Centre, which was established collaboratively between ETH Zurich and Singapore's National Research Foundation (FI 370074016) under its Campus for Research Excellence and Technological Enterprise programme. 